\title{
Significance of Body Mass Index in the Classification of PCOS: A Comparative Study in Northeast India
}

\author{
Swapna S Pillai ${ }^{1}$, Pranay K Phukan ${ }^{2}$, Prasanta Dihingia ${ }^{3}$
}

\begin{abstract}
Aim: To compare the clinical and biochemical characteristics of obese and lean women with polycystic ovary syndrome (PCOS). To confirm the significance of body mass index (BMI) in classifying PCOS.

Materials and methods: This was a comparative cross-sectional study done at Assam Medical College Hospital, Dibrugarh, Assam, in 1 year. Women who attended the hospital diagnosed to have PCOS based on Rotterdam's criteria were divided into lean and obese with BMI cut-off of 23. Clinical, anthropometric, and biochemical characteristics of both were compared. Body fat percentage was calculated using Harpenden skinfold calipers, and based on it, the lean group was found to have a normal weight, and obese subgroup with body fat percentage more than the cut-off.

Result: There was a significant difference in age, hirsutism score, and family history of diabetes and PCOS between the groups. The systolic blood pressure, serum cholesterol, and serum low-density lipoprotein (S.LDL) were statistically higher in the obese group. The normal weight obese group had features of the lean group, except for hyperandrogenism.

Conclusion: The lean and obese PCOS are two discrete groups. Obese patients have a more androgenic and atherogenic lipid profile, but both groups should be screened. Body mass index is the easily available method, but it has a chance of missing out on the normal weight obese PCOS. Clinical significance: Follow-up is needed in both the groups, and the normal weight obese group behaves as the lean PCOS group; hence, BMI can be continued to be used as the differentiating factor.
\end{abstract}

Keywords: Anthropometric, Body mass index, Body fat, Lean, Normal weight, Obese, Polycystic ovary syndrome.

Journal of South Asian Federation of Obstetrics and Gynaecology (2020): 10.5005/jp-journals-10006-1776

\section{INTRODUCTION}

Polycystic ovary syndrome (PCOS) is one of the most common endocrinopathies affecting approximately $4-12 \%$ of the reproductive age group women. ${ }^{1}$ Polycystic ovary syndrome was defined at a joint consensus meeting of the European Society of Human Reproduction and Embryology (ESHRE) and the American Society of Reproductive Medicine (ASRM), held in Rotterdam in May 2003. ${ }^{2}$ Polycystic ovary syndrome is mainly characterized by irregular menstruation with features of hyperandrogenism and polycystic ovaries on ultrasonography (USG) examination. The hyperandrogenism clinically manifests as hirsutism, acne, male-pattern baldness, hoarseness of voice, etc. The ovulatory dysfunction associated may present as menstrual disturbances such as oligomenorrhea, amenorrhea, rarely polymenorrhea, chronic anovulation, and infertility.

Infertility is said to be 10 times more common among the PCOS patient, and in India, it is one of the major problems faced by the reproductive age group women. ${ }^{3}$ Women with PCOS tend to have features of abnormal lipid profile, hyperinsulinemia, adverse reproductive outcomes, ${ }^{4}$ and later on, develop long-term metabolic disorders ${ }^{5,6}$ and increased association with the risk of ovarian, endometrial, and breast cancer. ${ }^{7}$ Thus, an early evaluation can reduce the burden of the disease.

The PCOS is said to have two phenotypes: the obese and the lean, the latter being a much less common presentation of the disease based on the BMI. The exact pathophysiology affecting the two groups is yet to be elaborated.

The BMI is the most commonly used anthropometric measurement for classifying individuals into groups. It is being \begin{tabular}{l}
\hline \hline 1,2Department of Obstetrics and Gynaecology, Assam Medical College, \\
Dibrugarh, Assam, India \\
3Department of Medicine, Diphu Medical College, Diphu, Assam, India \\
Corresponding Author: Pranay K Phukan, Department of Obstetrics \\
and Gynaecology, Assam Medical College, Dibrugarh, Assam, India, \\
Phone: +91 9435031732, e-mail: pranayp4@yahoo.com \\
How to cite this article: Pillai SS, Phukan PK, Dihingia P. Significance of \\
Body Mass Index in the Classification of PCOS: A Comparative Study in \\
Northeast India. J South Asian Feder Obst Gynae 2020;12(3):145-149. \\
Source of support: Nil \\
Conflict of interest: None
\end{tabular}

stated as a rather poor indicator of the percentage of body fat and has low sensitivity to determine excess adiposity. ${ }^{8}$ Even though applicable in the population level, BMI tends to misclassify at the individual level.

The association between BMI and body fat is not strong and has a significant difference with age, gender, and ethnicity. ${ }^{9}$ The peripheral body fat percentage is an important prognostic marker for metabolic complications. Hence, by using only BMI to classify PCOS, there is an inherent risk of overclassifying or underclassifying cases. Thus, a more specific method of measuring the body fat using the Harpenden skinfold caliper (CMS Instruments Ltd., London) has been used. Thus, we can identify the significance of each method in classifying PCOS.

Limited data were available from India regarding the clinical and biochemical differences among the lean and obese women having PCOS. In most of the available studies, BMI is taken as a

(0) The Author(s). 2020 Open Access This article is distributed under the terms of the Creative Commons Attribution 4.0 International License (https://creativecommons. org/licenses/by-nc/4.0/), which permits unrestricted use, distribution, and non-commercial reproduction in any medium, provided you give appropriate credit to the original author(s) and the source, provide a link to the Creative Commons license, and indicate if changes were made. The Creative Commons Public Domain Dedication waiver (http://creativecommons.org/publicdomain/zero/1.0/) applies to the data made available in this article, unless otherwise stated. 
measurement of body fat; therefore, here we compare the clinical and biochemical profile of obese and lean PCOS women and determine whether any significant difference is present along with the incorporation of body fat percentage assessment.

\section{Materials and Methods}

In the present comparative cross-sectional study, around 100 women in the age group of 14-40 years attending the Gynaecology OPD of Assam Medical College, Dibrugarh, between July 2018 and June 2019 were evaluated, and women who were diagnosed to have PCOS according to Rotterdam's criteria were included. Based on the BMI, PCOS is divided as lean PCOS $<23 \mathrm{~kg} / \mathrm{m}^{2}$ and obese PCOS $\geq 23$ $\mathrm{kg} / \mathrm{m}^{2}$, based on WHO classification of BMI for the Asian population. Here, we consider the overweight individuals also as obese PCOS. ${ }^{10}$ Considering $95 \%$ of confidence interval with a power of $90 \%$ and taking the findings of the study by Panda et al.'s reference, the sample size for the present study was calculated and rounded off to be 30 in each group. ${ }^{11}$ Of these, 30 consecutive cases of each lean and obese PCOS were studied with detailed history, clinical examination, and biochemical evaluation.

Women suffering from other causes of hyperandrogenism, hyperprolactinemia, hypogonadotropic hypogonadism, thyroid disorders, cases of premature ovarian failure, congenital adrenal hyperplasia, androgen-secreting tumor, Cushing's syndrome, uterine disorders (e.g., Asherman's syndrome, Mullerian agenesis), chromosomal anomalies, patients receiving medications known to affect carbohydrate metabolism, pregnancy, and lactation, women under oral contraceptive pills, women having ovarian cysts or tumors were excluded.

Approval from the ethics committee of the hospital was taken. The selected women were counseled and explained about the study, and the written and informed consent was taken.

Detailed sociodemographic history was taken followed by the clinical examination for signs of hyperandrogenism like hirsutism and acne and hyperinsulinemia like acanthosis nigricans. Hirsutism is graded according to the Ferriman-Gallwey scoring. Blood pressure and other anthropometric measurements such as height, weight, waist circumference, and hip circumference were measured. Waist-to-hip ratio was calculated. The BMI was calculated based on the formula of weight divided by the square of height in meter.

Harpenden skinfold calipers were used to measure the skinfold thickness from four sites: the biceps, triceps, subscapular, and suprailiac region, and the body fat indices such as fat percentage, fat mass, and lean body mass were calculated using the Durnin and Womersley (DW) equations. All the parameters were compared in both the groups. All skinfold measurements (i.e., triceps, biceps, suprailiac, and subscapular) should be picked up between the thumb and forefinger; pinch the skin and pull it away from the underlying muscle; apply caliper $1 \mathrm{~cm}$ from the ridge of the skin thus formed; take reading 3 seconds after the application of caliper, to standardize any effects produced by deformation of the tissue; do not remove your hold of the skinfold while the caliper is applied; record the average of three readings. The method of measuring the skinfold thickness is shown in Figure 1.

Based on the studies of Hortobágyi et al., ${ }^{12}$ the body fat percentage cut-off was taken to be $30 \%$, and a group of individuals whose BMI was in the normal range but whose peripheral body fat percentage was in the higher range was identified and called as the normal weight obese group.

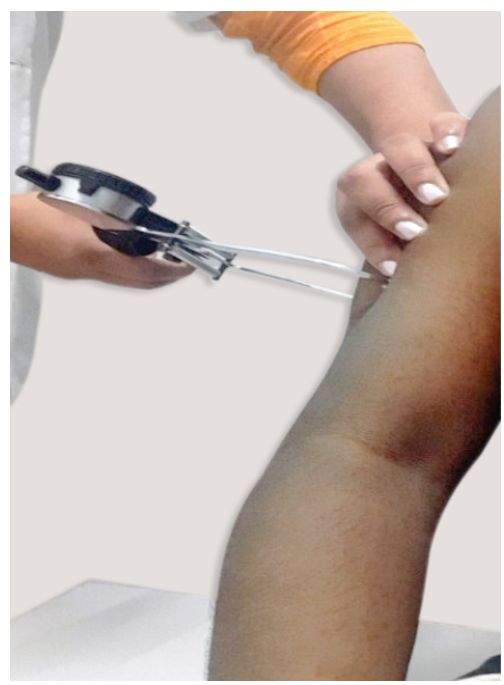

Fig. 1: Method of measuring skinfold thickness

In all the participants' thyroid profile, fasting blood glucose, postprandial blood sugar, $\mathrm{HbA1c}$, serum prolactin, serum antimullerian hormone (AMH), and lipid profile were done and compared among the groups. Pelvic USG for unmarried women and transvaginal USG for married women were also performed on days 9,10 , or 11 of the menses. Flowchart 1 shows an overview of the methodology.

The statistical analysis of data was performed using the computer program - Statistical Package for Social Sciences (SPSS for Windows, version 20.0., Chicago, SPSS Inc.) and Microsoft Excel 2010. Results on continuous measurements were presented as mean \pm standard deviation, and the two groups were compared using the Student's $t$ test. Discrete data were expressed as number (\%), and the two groups were analyzed using the chi-square test and Fischer's exact test (where the cell counts were $<5$ or 0 ). For all analyzes, the statistical significance was fixed at $5 \%$ level ( $p$ value $<0.05$ ).

The characteristics of having a significant difference between the obese and lean group were again compared with the normal weight obese group with a one-way ANOVA test with post hoc Tukey HSD test to identify significance between the three groups.

\section{Results}

All the women included in the study had complaints of menstrual irregularity. The majority of the women $(90 \%$ of lean and $83 \%$ of obese) were married and all of them had associated infertility. The mean age among the lean group was $24.17 \pm 4.74$, and among obese, it was $26.80 \pm 5.04$; there was a significant difference in the age distribution ( $p$ value: 0.04 ). None of the first-degree relatives of the lean group had significant family history, whereas the significant history of PCOS (40.00\%, $p$ value: 0.005$)$ and diabetes mellitus ( $36.67 \%, p$ value: 0.005$)$ was found in obese PCOS subjects. Regarding hyperandrogenism features, it was noticed that the obese group had a higher hirsutism score than lean $(12.97 \pm 4.81$ with $p$ value of $<0.001)$. There was no difference in the prevalence of menstrual irregularity, infertility, and acne and acanthosis nigricans in-between the two groups. The systolic blood pressure among the obese group was significantly raised from the lean group, but this difference was not seen in diastolic blood pressure (BP). Table 1 shows the clinical and anthropometric findings of lean and obese PCOS. 
Flowchart 1: Flowchart of methodology
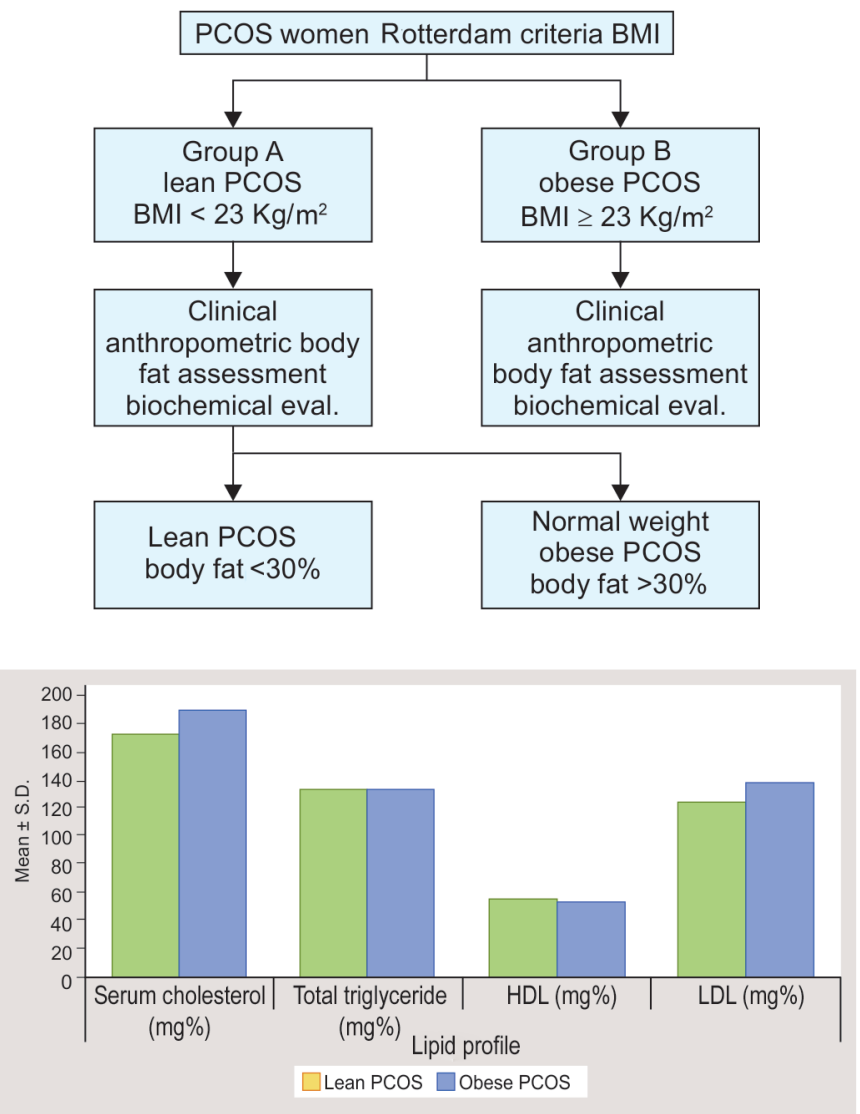

Fig. 3: Lipid profile of obese and lean PCOS

Table 1: Clinical and anthropometric features between the two groups

\begin{tabular}{llll}
\hline Characteristics & Lean PCOS & Obese PCOS & p value \\
\hline Age $^{\mathrm{b}}$ & $24.17 \pm 4.74$ & $26.80 \pm 5.04$ & $0.042^{\mathrm{d}}$ \\
Family history of diabetes $^{\mathrm{c}}$ & $2(6.67)$ & $11(36.67)$ & $0.005^{\mathrm{d}}$ \\
Family history of PCOS $^{\mathrm{c}}{ }^{\mathrm{c}}$ & $1(3.33)$ & $12(40.00)$ & $0.005^{\mathrm{d}}$ \\
Menstrual irregularity $^{c}$ & $30(100.00)$ & $30(100.00)$ & 1 \\
Infertility $^{c}$ & $27(90)$ & $25(83.3)$ & 0.4 \\
Hirsutism score $^{\mathrm{b}}$ & $9.57 \pm 2.43$ & $12.97 \pm 4.81$ & $0.001^{\mathrm{d}}$ \\
Acne $^{\mathrm{c}}$ & $4(13.33)$ & $7(23.33)$ & 0.31 \\
Acanthosis nigricans $^{c}$ & $3(10.00)$ & $4(13.33)$ & 0.68 \\
Systolic BP $(m m ~ H g)^{\mathrm{b}}$ & $116.43 \pm 5.65$ & $127.83 \pm$ & $<0.001^{\mathrm{d}}$ \\
& & 5.13 & \\
WHR $^{\mathrm{b}}$ & $0.80 \pm 0.02$ & $0.91 \pm 0.05$ & $0.001^{\mathrm{d}}$ \\
\hline
\end{tabular}

$\mathrm{BP}$, blood pressure; BMI, body mass index; WHR, waist-to-hip ratio; LDL, low-density lipoprotein; HDL, high-density lipoprotein

${ }^{a}$ Continuous variables are given as mean \pm SD, categorical variables are presented as number (percentage).

${ }^{\mathrm{b}}$ Family history among first-degree relatives

'Variables compared using the Student's $t$ test

${ }^{d}$ Variables compared using Chi-square test

$e_{p}$ value $<0.05$ is considered significant

The anthropometric measurement of obese PCOS group had higher values compared to the lean like in waist-to-hip ratio $(0.91 \pm 0.05, p$ value $<0.001)$, BMI $\left(31.19 \pm 2.60 \mathrm{~kg} / \mathrm{m}^{2}, p\right.$ value $<0.001)$, body fat percentage $(42.46 \pm 2.95 \%, p$ value $<0.001)$, and lean body mass $(44.60 \pm 3.08 \mathrm{~kg}, p$ value $<0.001)$. The BMl and

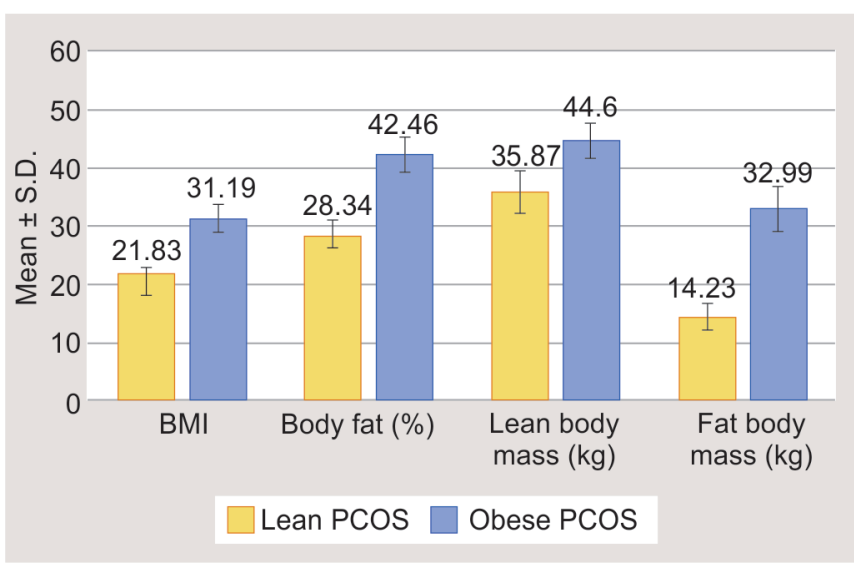

Fig. 2: Comparison of BMI and body fat composition

the body fat composition are shown in Figure 2. The mean serum cholesterol and serum LDL were raised in obese PCOS patients, whereas the rest of the lipid profile, thyroid, and blood sugar level was similar. Lean PCOS has a slightly higher level of serum HDL $(54.65 \pm 9.52, p$ value 0.247$)$. Figure 3 shows the lipid profile of the obese and lean PCOS.

Based on studies of Hortobágyi et al., ${ }^{12} 33 \%$ of the lean group was found to have body fat percentage more than the cut-off. This group had been called as the normal weight obese PCOS women. Analysis between the three groups was then done using the one-way ANOVA test with the characteristics having a significant difference between the obese and lean group, and subsequently, the post hoc Tukey's HSD test was done to confirm the significance. It was found that there was no significant difference between obese and normal weight obese groups based on the hirsutism score, but for the rest of the factors such as systolic BP, waist-to-hip ratio, BMI, body fat percentage, total cholesterol, and S.LDL, there was significant difference between obese and normal weight obese. The test data are shown in Table 2.

\section{Discussion}

From the study, it was seen that two discrete groups exist among PCOS women - the obese and the lean group. The majority of elderly age group PCOS women belong to the obese category and rarely to the lean group. Obese PCOS is associated with a family history of either diabetes or PCOS, and none of the first-degree relatives of lean PCOS had any such history. Thus, it strengthens the genetic relation of PCOS.

Menstrual irregularity was the most common presentation seen in all patients, and the prevalence of the different patterns of menstrual cycles did not differ between obese and lean women; thus, the anthropometric findings rarely define the prevalence of menstrual irregularity. It was already shown in previous studies. ${ }^{13-15}$ Oligomenorrhea is the most common menstrual irregularity followed by amenorrhea. Very rarely, patients are presented with menorrhagia and polymenorrhea. Among unmarried females, significant findings were of hirsutism, acne, and weight gain.

Infertility was the second most common presenting complaint in our study with $27(90 \%)$ of lean and $26(86.67 \%)$ of obese women having it. The prevalence of infertility was high in our study as the women from the lower socioeconomic strata attend the OPD because of fertility issues rather than for the symptoms of PCOS. 
Significance of Body Mass Index in the Classification of PCOS: A Comparative Study in Northeast India

Table 2: Comparison using the one-way ANOVA test and post hoc Tukey HSD test

\begin{tabular}{lcccc}
\hline Variable & Lean PCOS & Normal-weight obese PCOS & Obese PCOS & $p$ value \\
\hline BMI & $21.78 \pm 1.14^{\mathrm{a}}$ & $21.85 \pm 1.18$ & $31.19 \pm 2.6$ & 0.007 \\
Hirsutism score & $9.45 \pm 2.42^{\mathrm{a}}$ & $10.9 \pm 1.6^{\mathrm{b}}$ & $12.97 \pm 4.81$ & $<0.01$ \\
Systolic BP & $116.10 \pm 5.89^{\mathrm{a}}$ & $116.2 \pm 5.37^{\mathrm{b}}$ & $127 \pm 5.13$ & $<0.001$ \\
Body fat\% & $26.76 \pm 1.64^{\mathrm{a}}$ & $31.5 \pm 1.89^{\mathrm{b}}$ & $42.46 \pm 2.95$ & $<0.001$ \\
S. cholestrol & $170.81 \pm 6.48^{\mathrm{a}}$ & $169.8 \pm 6.55^{\mathrm{b}}$ & $189.37 \pm 14.35$ & $<0.001$ \\
S. LDL & $124.10 \pm 5.76^{\mathrm{a}}$ & $123.3 \pm 5.5^{\mathrm{b}}$ & $139.73 \pm 7.77$ & $<0.001$ \\
\hline
\end{tabular}

Continuous variables are given as mean $\pm \mathrm{SD} ; p$ value less than 0.05 is considered significant

${ }^{a}$ Comparison between lean and obese PCOS is significant

${ }^{b}$ Comparison between normal weight obese and obese PCOS is significant

The prevalence of infertility in women with PCOS varies between 70 and $80 \% .{ }^{16}$ The influence of PCOS phenotype, lean and obese, on female fertility remains poorly comprehended, and the present study shows that even lean PCOS has a high prevalence of infertility.

Obese women with PCOS had a high hirsutism score than lean. Lean PCOS when presented with hirsutism only had mild grade never moderate or severe grade, whereas the obese group had women of moderate and severe grade. Higher hyperandrogenism was associated with women of high BMI. Sachdeva et al. also showed a higher hirsutism score in the obese group. ${ }^{17}$

Even though none of the patients was found to be hypertensive, it was seen that obese PCOS had a higher systolic BP; hence, proper evaluation is needed for early detection of hypertensives, especially in the obese group.

The previous studies had shown that obese PCOS is associated with central adiposity, ${ }^{14}$ and the present study has also shown similar results with the obese group having a higher waist-to-hip ratio. The mean body fat percentage of the obese group (42.46 \pm $2.95 \%)$ was higher than the lean group $(28.34 \pm 2.84 \%)$, yet the debate keeps on continuing whether obesity leads to PCOS or vice versa.

The mean serum cholesterol of the obese group was found to be higher than the lean group $(189.37 \pm 14.35$ vs. $172.04 \pm 5.97$ $\mathrm{mg} \%$ with $p$ value $<0.001$ ), and the difference in LDL was also found to be significant with the obese group having a higher value. However, the lean group has a higher value of the good cholesterol, HDL. Several Indian studies have shown both the groups having a significant difference, ${ }^{18}$ and other studies had not found any significance between the two groups when compared to weightmatched healthy adults. ${ }^{11}$ Thus, in general, it can be inferred that obese PCOS is said to have a more atherogenic lipoprotein pattern, but we cannot rule out the fact that even lean PCOS women have deranged lipid profile compared to normal control. Thus, it can be recommended that both lean as well as obese PCOS patients, the lipid profile should be evaluated for early detection and prevention of future cardiac complications.

Based on the ANOVA test done between the three groupslean, obese, and normal weight obese, it was clear that even though a small group of normal weight obese PCOS was there, it was not different from the lean group except in the point of hyperandrogenism. Thus, findings support the continuation of the use of BMI for differentiating the PCOS women into lean and obese groups. Further studies are needed to identify whether any significant difference exists in the aspect of insulin resistance, hyperandrogenism, cardiovascular risk factors, or fertility between the normal weight obese PCOS and lean PCOS group.

The present study had some limitations. First, the data were only from patients attending one hospital; second, the basic characteristics of economic and nutritional status were not matched; third, other methods of evaluation for body fat percentage DEXA scan could not be used due to the economic constraints.

\section{Conclusion}

Thus, in conclusion, we can state that obese PCOS had a more androgenic and atherogenic profile compared to lean with strong family history and increased predisposition for hypertension. Body mass index can be continued as an indicator of obesity especially in the absence of facilities to measure the actual body fat percentage. At the same time, the need for follow-up studies on the normal weight obese group is warranted.

\section{Clinical Significance}

Follow-up is needed in both the groups, and the normal weight obese group behaves as the lean PCOS group; hence, BMI can be continued to be used as the differentiating factor.

\section{Ethical Considerations}

The research protocol was approved by the Institutional Ethics Committee before the study began [No. AMC/EC/PG/2596].

\section{InFORMED CONSENT}

All the participants gave written informed consent before the study began.

\section{Author Contributions}

Swapna S Pillai: data collection, analysis, protocol development, and manuscript writing; Pranay K Phukan: data analysis, protocol development, and manuscript writing; Prasanta Dihingia: data analysis and protocol development.

All authors reviewed the manuscript and agreed to be accountable for all aspects of the work.

\section{Data Availability}

The database generated during the current study is available from the corresponding author on a reasonable request. 


\section{Manufacturer's Name}

Harpenden skinfold caliper, CMS Instruments Ltd., London

\section{ACKnOWLedgments}

The study was done in collaboration with the ICMR-funded study named "PHEnotyping Northeast INDian Young type II diabetes (PHENOEINDY-2)" done in the Medicine Department of Assam Medical College.

\section{References}

1. Majumdar A, Singh TA. Comparison of clinical features and health manifestations in lean vs. obese Indian women with polycystic ovarian syndrome. J Hum Reprod Sci 2009;2(1):12-17. DOI: 10.4103/0974-1208.51336.

2. Rotterdam ESHRE/ASRM-Sponsored PCOS Consensus Workshop Group. Revised 2003 consensus on diagnostic criteria and longterm health risks related to polycystic ovary syndrome. Fertil Steril 2004;81(1):19-25. DOI: 10.1016/j.fertnstert.2003.10.004.

3. Hart R, Doherty DA. The Potential implications of a PCOS diagnosis on a woman's long-term health using data linkage. J Clin Endocrinol Metab 2015;100(3):911-919. DOI: 10.1210/jc.2014-3886.

4. Boomsma CM, Eijkemans MJC, Hughes EG, et al. A meta-analysis of pregnancy outcomes in women with polycystic ovary syndrome. Hum Reprod Update 2006;12(6):673-683. DOI: 10.1093/humupd/ dml036.

5. Dunaif A. Insulin action in the polycystic ovary syndrome. Endocrinol Metab Clin North Am 1999;28(2):341-359. DOI: 10.1016/S08898529(05)70073-6.

6. Dahlgren E, Johansson S, Lindstedt G, et al. Women with polycystic ovary syndrome wedge resected in 1956 to 1965: a long-term follow-up focusing on natural history and circulating hormones. Fertil Steril 1992;57(3):505-513. DOI: 10.1016/S0015-0282(16)54892-4.

7. Balen A. Polycystic ovary syndrome and cancer. Hum Reprod Update 2001;7(6):522-525. DOI: 10.1093/humupd/7.6.522.

8. Okorodudu DO, Jumean MF, Montori VM, et al. Diagnostic performance of body mass index to identify obesity as defined by body adiposity: a systematic review and meta-analysis. Int J Obes (Lond) 2010;34(5):791-799. DOI: 10.1038/ijo.2010.5.

9. Meeuwsen S, Horgan GW, Elia M. The relationship between BMI and percent body fat, measured by bioelectrical impedance, in a large adult sample is curvilinear and influenced by age and sex. Clin Nutr 2010;29(5):560-566. DOI: 10.1016/j.clnu.2009.12.011.

10. WHO Expert Consultation. Appropriate body-mass index for asian populations and its implications for policy and intervention strategies. Lancet 2004;363(9403):157-163. DOI: 10.1016/S01406736(03)15268-3.

11. Panda S, Rout P, Chandra C. A case control study of role of lipid profile in polycystic ovarian syndrome: is there any role in non-obese polycystic ovary syndrome? Int J Reprod Contracept Obstet Gynecol 2016;5(6):1981-1984.

12. Hortobágyi T, Israel RG, O'Brien KF. Sensitivity and specificity of the Quetelet index to assess obesity in men and women. Eur J Clin Nutr 1994;48(5):369-375.

13. Panidis D, Tziomalos K, Papadakis E, et al. Associations of menstrual cycle irregularities with age, obesity and phenotype in patients with polycystic ovary syndrome. Hormones 2015;14(3):431-437.

14. Liou TH, Yang JH, Hsieh $\mathrm{CH}$, et al. Clinical and biochemical presentations of polycystic ovary syndrome among obese and nonobese women. Fertil Steril 2009;92(6):1960-1965. DOI: 10.1016/j. fertnstert.2008.09.003.

15. Boumosleh JM, Grundy SM, Phan J, et al. Metabolic concomitants of obese and nonobese women with features of polycystic ovarian syndrome. J Endocr Soc 2017;1(12):1417-1427. DOI: 10.1210/js.201700323.

16. Yuan C, Liu X, Mao Y, et al. Polycystic ovary syndrome patients with high BMI tend to have functional disorders of androgen excess: a prospective study. J Biomed Res 2016;30(3):197-202.

17. Sachdeva G, Gainder S, Suri V, et al. Obese and non-obese polycystic ovarian syndrome: comparison of clinical, metabolic, hormonal parameters, and their differential response to clomiphene. Indian J Endocrinol Metab 2019;23(2):257. DOI: 10.4103/ijem.IJEM_637_18.

18. Akshaya S, Bhattacharya R. Comparative study of metabolic profile of women presenting with polycystic ovary syndrome in relation to body mass index. Int J Reprod Contracept Obstet Gynecol 2016;5(8):2561-2565. 